\title{
Risk factors associated with peristomal skin complications: Integrative literature review
}

\author{
Blessy John*1, Mi-Yeon Kim², Daniel Forgrave ${ }^{2}$ \\ ${ }^{1}$ Department of Nursing, National Center for Cancer Care \& Research, Hamad Medical Corporation, Doha, Qatar \\ ${ }^{2}$ University of Calgary in Qatar, Doha, Qatar
}

Received: November 22, 2018

DOI: $10.5430 /$ jnep.v9n7p82
Accepted: March 6, 2019

Online Published: March 29, 2019

\begin{abstract}
Surgical intervention of colorectal cancer often includes formation of ostomy. Irrespective of the type of stoma, many ostomy patients suffer from peristomal skin complications. Identifying risk factors related to peristomal skin complications is one of the crucial factors in maintaining peristomal skin health. The purpose of this integrative review is to identify the risk factors associated with the development of peristomal skin complications. Whittmore and Knafl's (2005) framework for integrative literature review guided this study. The results of this systematic literature review showed risk factors for developing peristomal skin complications are multidimensional. Leakage of stoma output, type and structure of stoma, stoma site marking and nature of surgery, ostomy education, ostomy appliances, mechanical trauma, and demographic factors are the most commonly identified risk factors in the development of peristomal skin complications. The development of peristomal skin complications is closely associated with skin contact with ostomy effluent. In all settings ostomy patients requires specialized care and management to prevent development of peristomal skin complications and promote quality of life. The findings of this focused study suggest that prevention and early identification of risk factors related to peristomal skin complications are very important.
\end{abstract}

Key Words: Peristomal skin complications (PSCs), Stoma, Ostomy, Colostomy, Ileostomy, Urostomy

\section{INTRODUCTION}

Globally, the third most common cancer is colorectal cancer (CRC). It is the fourth leading cause of cancer-related death accounting for 1.4 million incidences and almost 700,000 deaths in 2012. ${ }^{1]}$ The incidence of CRC in the Arabian Gulf region in general is lower than that of other countries. Nonetheless, in countries such as Oman, Qatar, and Saudi Arabia, the incidence rate of CRC in people under 40 years is comparatively high. ${ }^{[2]} \mathrm{CRC}$ is the most common cancer among the male population in Qatar, ${ }^{[3]}$ in which approximately $20.8 \%$ of these are Qatari. ${ }^{[4]}$ One of the major treatments of CRC is surgical intervention. The surgical man- agement of CRC often results in the formation of ostomy. Based on the statistics of the colorectal department in Hamad Medical Corporation (HMC), the total number of ostomy surgeries performed between July 2015 and July 2016 in Hamad General Hospital was 55. Of these, 49 cases were related to colorectal cancer (A. Ben, personal communication, March 5, 2017), and the major issue facing by those patients are peristomal skin complications (PSCs). PSCs are types of different dermatoses affecting the skin immediately surrounding a stoma. ${ }^{[5]}$ Individuals with ostomy face a number of skin complications. Identifying risk factors related to PSCs is one of the crucial factors that influences post stoma

\footnotetext{
*Correspondence: Blessy John; Email: blessy85222@yahoo.com; Address: Department of Nursing, National Center for Cancer Care \& Research, Hamad Medical Corporation, P.O Box 3050, Doha, Qatar.
} 
adjustment in individuals with ostomy. According to Meisner, Lehur, Moran, Martins, and Jemec, ${ }^{[6]}$ PSCs are the most widely recognized problem after the formation of a stoma. Stoma and peristomal skin care are challenging experience for patients and their families or caregivers. Peristomal skin issues following intestinal ostomy surgery can compromise the quality of life of ostamates and lead to limitations in physical, psychological, and social wellbeing.

As one of Qatar's health strategy goals is preventive health care, the findings of this study have important implication for the care of ostomy patients living in Qatar. Reduction in the incidences of PSCs from effective management of risk factors would empower ostomy patients to enhance self-care and promote a sense of wellness.

\subsection{Background}

\subsubsection{Stoma}

Creation of a stoma is an essential part of many abdominal surgical interventions. An ostomy or stoma is a surgically created opening whose meaning is derived from the Greek word meaning opening or mouth. ${ }^{[7-9]}$ In appearance, a normal stoma appears red, wet, and is warm to the touch. ${ }^{[10]}$

\subsubsection{Peristomal skin complications}

Peristomal skin is the area around the stoma where an ostomy appliance adheres. ${ }^{[11]}$ Healthy peristomal skin presents without redness, loss of epidermis, itchiness, warmth, or pain. Peristomal skin health is dependent on a number of factors, including the ability of the body to interact with skin barriers and adhesive materials, type of stoma, type of appliances, and ostomy dressing itself. ${ }^{[12]}$ PSCs are different types of inflammatory or infectious conditions affecting the skin immediately surrounding the stoma. ${ }^{[13]}$ Common PSCs affecting ostomy patients are dermatitis, skin stripping, encrustations, psoriasis, pyoderma gangrenosum, folliculitis, infections, mucocutaneous separation, over granulation, excoriation, maceration, and trauma. ${ }^{[13,14]}$

The incidence rate of PSC is substantial and varies in magnitude across countries. Ratliff ${ }^{[15]}$ reported that $47 \%(n=89)$ of North American participants in his study were affected with stoma-related skin problems such as irritant dermatitis, mechanical injury, candida infection, and pyoderma gangrenosum. A similar finding was reported by Erwin-Toth, Thompson, and Davis ${ }^{[16]}$ who found that $61 \%(n=733)$ of their study participants from North America experienced problems with their peristomal skin, yet only one half of these participants were able to correctly recognize their skin problems as attention- requiring conditions, such as irritant contact dermatitis, mechanical trauma, allergic contact dermatitis, and infection. Similarly, in a Danish cross sectional

Published by Sciedu Press study, Herlufsen et al. ${ }^{[17]}$ reported that $45 \%$ of their participants $(n=202)$ also experienced peristomal skin issues, such as erosion, maceration, erythema, dermatitis, foliculitis, excoriation, eczema, psoriasis, and pyoderma gangrenosum. The effect of peristomal skin damage brings stoma-related pain and limits patients' mobility. ${ }^{[18]}$

\subsubsection{Impact of peristomal skin complications}

Research has shown that ostomy surgery in general initially reduces and significantly affects health-related quality of life (HQOL) over time. ${ }^{[19-23]}$ According to Dabirian, Yaghmaei, Rassouli, and Tafreshi, ${ }^{[24]}$ nine main challenges that ostomy patients face in relation to their quality of life (QOL) are physical problems, psychological problems, limited social and family relationships, economic challenges, nutritional issues, limited physical activity, restricted travel, disrupted religious rituals, and sexual issues. However, a limited number of studies have focused on the adjustment of patients who experience PSCs.

Many studies have focused on identification of causes and treatment of PSCs, a limited amount of research has explored the QOL of patients with PSCs. ${ }^{[25]}$ It is plausible to assume that PSCs may have a negative impact on QOL due to leakage, odor, and non-adherence of appliances. ${ }^{[26]}$

\subsection{Research question}

What are the risk factors associated with the development of peristomal skin complications?

\section{Methods}

An integrative literature review of primary studies was chosen to identify risk factors associated with peristomal skin complications. This design was chosen because it develops new knowledge regarding the reviewed issue. ${ }^{[27]}$ An integrative literature review is a study method in which data are collected from primary sources, which provide comprehensive knowledge and applicability of particular topic. ${ }^{[28]}$ An integrative literature review is "a specific review method that summarize past empirical or theoretical literature to provide a more comprehensive understanding of a particular phenomenon or health care problem". According to Whittemore and Knafl, ${ }^{[29]}$ an integrative literature review includes different experimental and non-experimental studies, and plays an important role in evidence-based nursing. The conceptual framework that guides this study was developed by Whittemore and Knafl, and encompasses five steps: problem identification, literature search, data evaluation, data analysis, and presentation of conclusions. 


\subsection{Problem identification}

The clear identification of the problem and the purpose of the review are the initial focus of any review. ${ }^{[30]}$ Problems addressed by this review have previously been identified as an increased incidence of PSCs and its negative impact on patients' QOL.

\subsection{Literature search}

A computer-based literature search was performed using key terms to explore different electronic databases including the Cumulative Index of Nursing and Allied Health Literature (CINAHL), Web of Science, Embase, and PubMed. Key terms used in the search were peristomal skin, colostomy, ileostomy, urostomy, ileal conduit, stoma, ostomy, risk*, effect*, impact*, influen*, consequence*, challeng*, complicat*, and barrier*. To obtain more specific information regarding PSCs, the key terms were combined with the Boolean operators AND or OR.

The inclusion criteria were primary studies published in scholarly peer reviewed journals of quantitative, qualitative, or mixed methods, and written in English. The exclusion criteria were grey literature, opinion or editorial publica- tions, review articles, unpublished manuscripts, as well as studies involving participants with multiple co-morbidities. The search was limited to studies published between 2006 and June 2017. From the search process, 563 articles were obtained for possible inclusion in this review.

\subsection{Data evaluation}

The initial search process yielded 563 articles. Following the application of limiters, 419 articles remained for possible inclusion. After the application of inclusion and exclusion criteria, 262 articles remained. After removing duplicates, 156 articles remained. The review of the title and abstract of 156 articles for relevancy eliminated 81 studies. The full texts of the remaining 75 articles were examined for relevancy for this review. Fifty-seven articles were eliminated, as they were not relevant to the topic. A manual search of the reference lists of the 18 remaining articles was performed, and an additional two studies were included because these articles were relevant to the research topic, leaving as final count of 20 articles. The studies included in the current integrated review consist of 18 quantitative studies and two qualitative studies (see Figure 1).

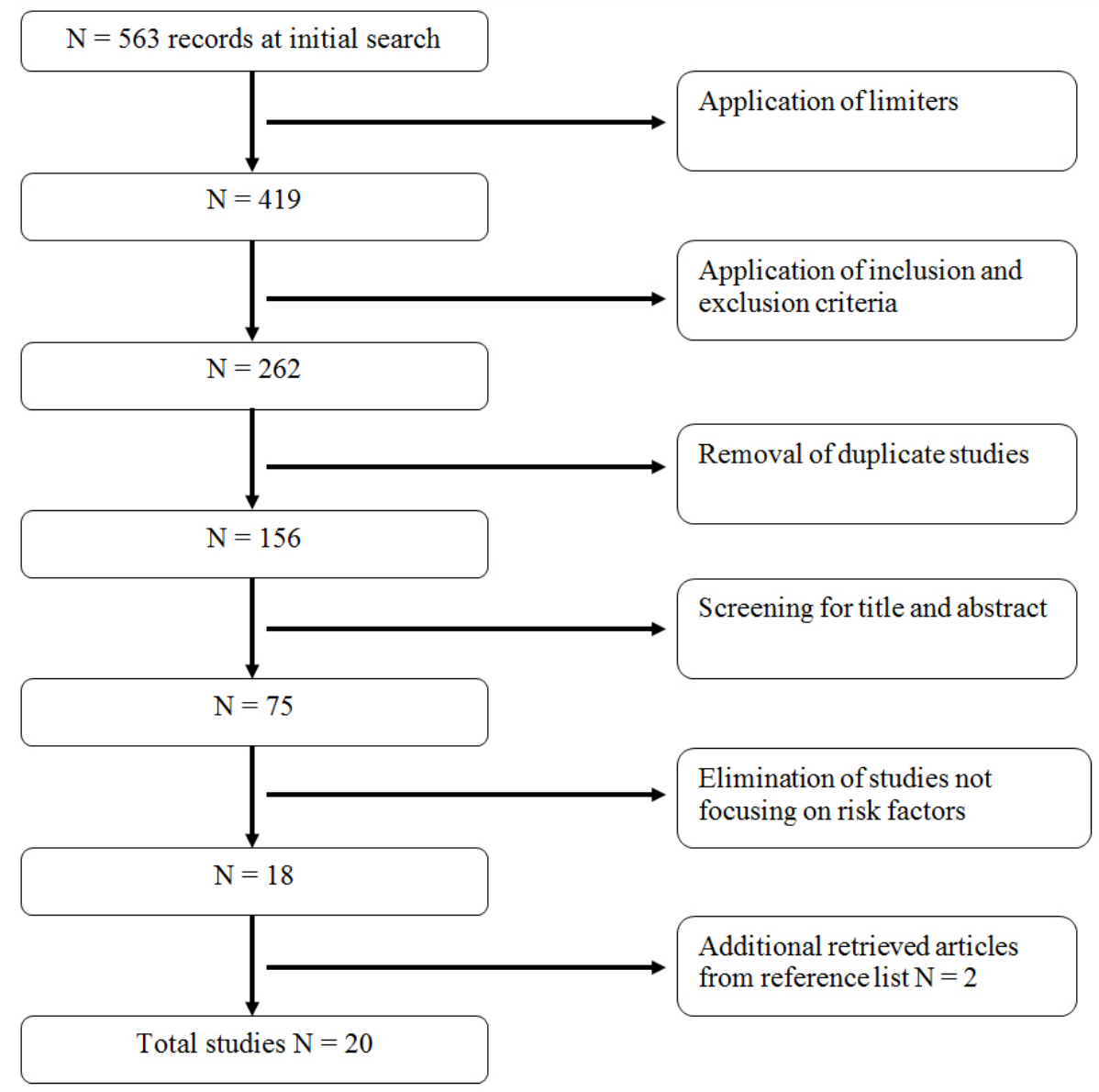

Figure 1. Flow chart of search process 


\subsection{Quality appraisal}

Evaluating the quality of studies included in this literature review was performed using two different critical appraisal tools. The qualitative studies were appraised with the Critical Appraisal Skills Program (CASP) and the quantitative studies were appraised with an adapted version of the Johns Hopkins Nursing Evidence-Based Practice Research Evidence Appraisal Tool.

\subsection{Data analysis}

Data analysis is done by categorizing, summarizing, and checking the level of evidence of each selected study, using tables, and conceptual maps. According to Whittemore and Knafl, ${ }^{[29]}$ the data analysis of literature reviews requires that data from primary sources be ordered, coded, categorized and summarised into a combined and joined summary about the research issue. They state that the goals of the data analysis stage are complete and unbiased explanation of primary data along with an innovative synthesis of the evidence. Data analyses of fundamental sources are examined in detail to extract data that could be used to identify themes and to conceptualize high-level abstraction. A literature review matrix was developed to display coded data, which were iteratively compared across studies to extract themes, discern relationships, and integrate data.

\subsection{Presentation}

According to Whittemore and Knafl, ${ }^{[29]}$ data can be presented in table or diagrammatic form. Therefore, based on the comprehensive review of the literature, a new conceptual model was developed to present the themes of this integrative literature review (see Figure 2).

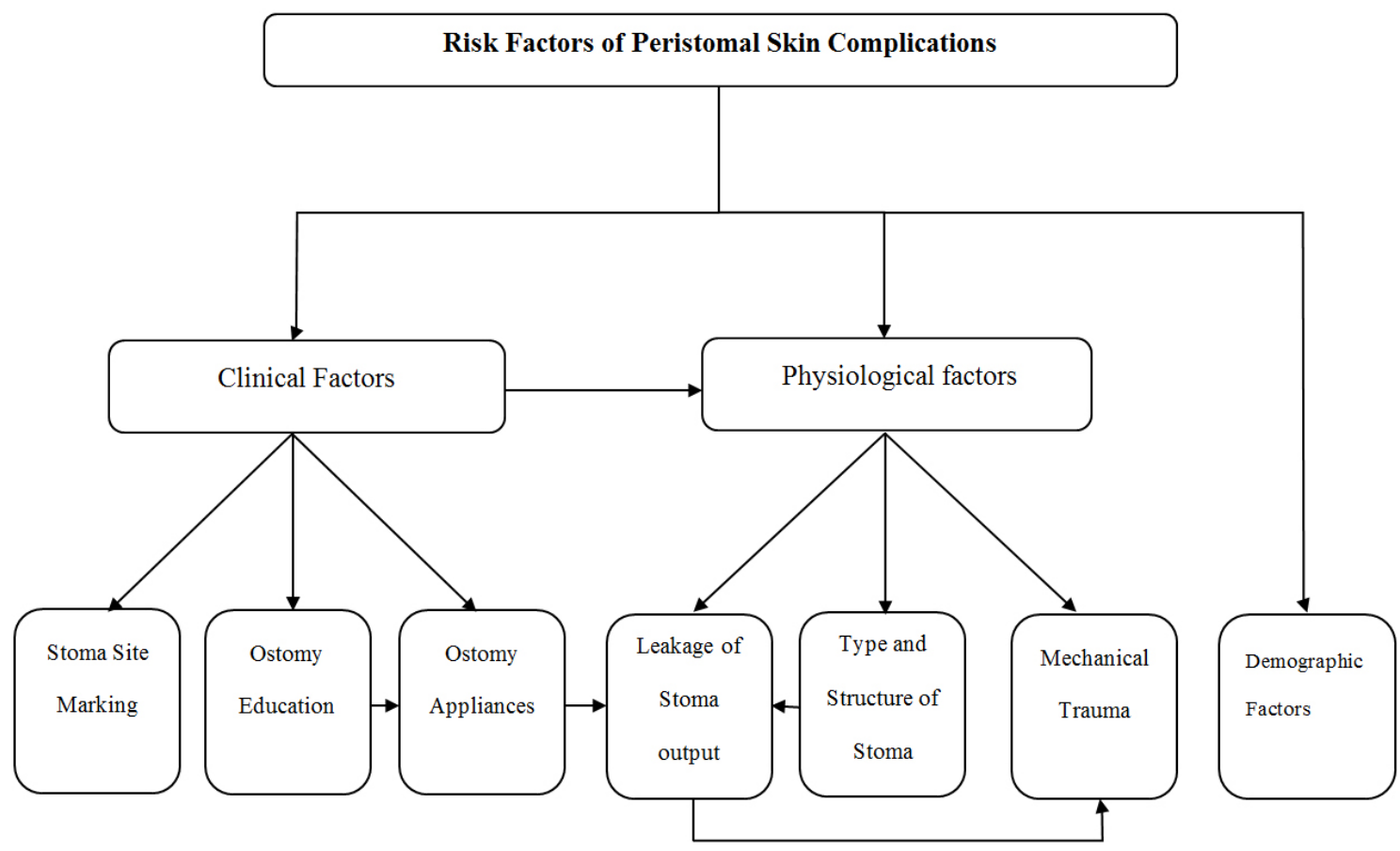

Figure 2. Conceptual model of risk factors of peristomal skin complication

\section{RESUlts}

The results of the analysis revealed seven core themes related to the risk factors of PSCs. The identified core themes are categorized into clinical and physiological, , and demographic factors. Clinical factors included: stoma site marking, ostomy education, and ostomy appliances. Physiological factors included: leakage of stoma output, type and structure of stoma, and mechanical trauma (frequent removal of skin barrier). Demographic factors are another core theme which includes age, gender, and Body Mass Index (BMI).

\subsection{Leakage of stoma output}

One of the most common risk factors of PSCs is the leakage from stomas. The leaky output leads to a poor fitting of appliances, which contributes to $61 \%$ of PSC cases. ${ }^{[30]}$ The contact between ostomy output and peristomal skin increases the risk of PSCs, as the effluent excoriates the skin. ${ }^{[30-33]}$ The contact of skin with ostomy effluent is the main cause of the development of fecal erosion, maceration, erythema, and contact dermatitis. ${ }^{[17]}$ An exploratory study showed that the detachment of stoma appliances and the subsequent skin maceration resulting from severe stoma leakage are signifi- 
cantly correlated $(p=.007)$. The management of PSCs such as skin maceration creates a huge challenge for participants and significantly lowers all aspects of their QOL. ${ }^{[34]}$ People with ostomies reported that stoma leakage is one of the main causes of altered social and psychological adjustment due to smell and soiling. ${ }^{[35]}$ Thus, it appears that participants with peristomal skin irritation were more likely to have frequent leakage than were those without skin irritation. ${ }^{[36]}$

\subsection{Type and structure of stoma}

Another risk factor of PSCs is the type of ostomy the individual is fitted with. ${ }^{[17]}$ The types of stomas that appear to increase the risk of PSCs are ileostomy, urostomy, end, and loop stomas. Patients with ileostomy seem to suffer from more skin complications in comparison to patients with other type of stomas. ${ }^{[15,32,37-39]}$ Skin excoriation has been reported as a common PSC among individuals with ileostomies. ${ }^{[30,37]}$ Although the evidence is unclear, the likely etiology is that the output from an ileostomy is more liquidy and acidic, and therefore, the output is more corrosive to the skin. ${ }^{[40]}$ Ratliff $^{[15]}$ reported that PSCs such as chemical irritant dermatitis and mucocutaneous separation are more common in people with ileostomies than with colostomies or an ileal conduit. Urostomy is the second most-common risk factor for developing PSCs. ${ }^{[17,36]}$ This is due to high water content and other urinary elements present in urinary output, which increases the onset of PSCs when in contact with the peristomal skin. ${ }^{[32]}$

Loop and end stomas are two of the main types of stomas created, and of these, a higher incidence of PSCs is observed with the loop type. Patients with loop ileostomy and loop colostomy seem to develop more skin problems than patients with end stomas. ${ }^{[38]}$ However, the evidence is conflicting with regard to the higher incidence of PSCs in people with loop stomas. For instance, Jayarajah, Samaresekara and Samarasekera ${ }^{[37]}$ reported that a higher rate of PSCs was observed among study participants who had end stomas in comparison to loop stomas. Similarly, Sung, Kwon, and Park $^{[33]}$ stated that the incidence of irritant contact dermatitis, hyperplasia, and peristomal hernia is significantly higher in people with end stoma than loop stoma. In addition, the rate of PSCs seems to escalate to the maximum when the construction of an end stoma occurs in patients with ileostomy (52.9\%). Still, some studies dispute the existence of a relationship between PSCs and ostomy type. ${ }^{[41,42]}$

The structure of a stoma, i.e. shape and height, also seems to influence the development of PSCs. ${ }^{[39]}$ Evidence suggests that oval- shaped stomas are the most common contributing factor for the development of skin issues in all types of ostomies during the initial three- month period. ${ }^{[38]}$ However, the findings are conflicting, as some authors claim that rather than oval-shaped stomas, circular-shaped stomas contribute more to the development of PSCs. ${ }^{[15]}$

An adequate height of the stoma is necessary to reduce the rate of stoma-related complications. Persson, Berndtsson, Carlsson, Hallén, and Lindholm ${ }^{[39]}$ found that a low stomal height has a significant association with problematic peristomal skin. For instance, a height of an ileostomy stoma lower than $20 \mathrm{~mm}$ and a colostomy stoma lower than $5 \mathrm{~mm}$ are risk factors for developing skin problems. However, studies selected in this integrative review did not elaborate on reasons or mechanisms underlying the relationship between the height of the stoma and the development of PSCs.

\subsection{Stoma site marking and nature of surgery}

The nature of surgery may further contribute to the development of PSCs. Stomal and peristomal complications, such as early skin irritation, are more frequently observed among patients who have undergone an emergency ostomy surgery, in comparison to patients who have chosen to have elective surgery. ${ }^{[38]}$ The absence of a preoperative stoma marking is another contributing factor for developing PSCs. ${ }^{[43]}$ In both elective and emergency surgery cases, a lack of pre-operative stoma marking may further increase the risk of developing PSCs after ostomy surgery. ${ }^{[4]}$ Preoperative stoma marking helps to contain the location of a stoma to within the rectus muscle, which is necessary to avoid the incidence of peristomal hernias, ${ }^{[33]}$ and to reduce the occurrence of other PSCs. ${ }^{[41]}$ Patients who have had emergency ostomy surgery are more likely to develop early skin irritation than patients who have had elective ostomy surgery. ${ }^{[44]}$ Patients who have had a pre-operative stoma marking encounter significantly less incidence of early skin irritation and dermatitis than patients without a stoma marking. ${ }^{[44]}$

\subsection{Ostomy education}

Stoma care education by wound and ostomy care nurses (WOCN) is another factor that may influence the development of PSCs. A lack of awareness and recognition of early signs and symptoms of stoma related complications contributes to the increased risk of PSCs. Ostomy education and regular follow up are necessary interventions to enable ostomates to recognize skin problems early and to learn about preventive measures for preservation of optimal peristomal skin health. ${ }^{[33,37,41,42]}$ Alenezi and Mansour ${ }^{[45]}$ showed that PSCs are more common in patients who did not receive proper stoma care education. More specifically, patients who did not receive preoperative stoma education reported more problems with skin irritation and severe leakage problems compared to those who received preoperative ostomy ed- 
ucation. ${ }^{[34]}$ Furthermore, the importance of including the caregivers, e.g., spouse, in ostomy education is evident in studies that showed an increased incidence of PSCs in the absence of spousal support with care of ostomy and appliances. ${ }^{[46]}$ Without ostomy education, patients did not know how to adjust their ostomy appliances properly for a better fit or choose appropriate appliances that are better suited to prevent leakage. Furthermore, a lack of regular follow up by ostomy care nurse meant patients did not have adequate knowledge or skills necessary to identify leakage and skin problems after having stoma surgery. ${ }^{[39]}$ Education by WOCN about proper pouching technique is important because leakage is often caused by incorrect pouching. Without the information about pouching technique, patients may not be able to avoid effluent coming into contact with the skin in order to prevent peristomal skin complications. ${ }^{[33]}$ When patients receive proper pre- and post-operative stoma care education, especially where there is no advanced ostomy care facility available, a significant reduction in the development of PSCs has been observed. ${ }^{[39]}$ Therefore, adequate information regarding ostomy and peristomal skin care is necessary to reduce the risk of developing PSCs.

\subsection{Ostomy appliances}

The type of ostomy appliances influences the development of PSCs. Møller Kruse and Storling ${ }^{[35]}$ suggested that different types of appliances are associated with different levels of detachment and leakage, which is a major risk factor of PSCs. Based on the outward shape, the base plate is categorized into convex (curved) and concave (flat) surface. ${ }^{[4]}$ Both concave and flat base plates can consist of either solid or soft flexible skin barriers. PSCs are more common in patients who use appliances with traditional flat skin barriers in comparison to appliances with flexible skin barriers. ${ }^{[48]}$ On the other hand, appliances such as a highly flexible wafer, seem to protect peristomal skin by reducing the risk of leakage and absorbing excess moisture from peristomal skin. ${ }^{[17]}$

Convexity of the base plate is another factor that increases the rate of PSCs. However, evidence on characteristics of the base plate is conflicting. For example, Ratliff ${ }^{[15]}$ found a higher incidence of PSCs was reported by patients who wore convex base appliances in comparison to patients who wore flat appliances. In contrast, patients who used a flat base appliance experienced more PSCs in comparison to patients who used an appliance with a convex base. In addition, convex stoma appliances helped to reduce the incidence of leakage, ${ }^{[42]}$ and reduce the incidence of mucocutaneous separation between the skin of the abdominal wall and the stoma. ${ }^{[43]}$ Another factor that influences the onset of PSCs is the nature of the base plate of the ostomy bag. The base plate

Published by Sciedu Press of an ostomy bag must be skin-friendly and have good adherence ability to prevent effluent from coming into contact with skin. ${ }^{[17,49]}$

\subsection{Mechanical trauma: Frequent removal of skin bar- rier}

Studies report that frequent removal of base plates (i.e. skin barrier) could impact the development of PSCs, and infrequent removal of base plates could reduce the development of peristomal skin irritation. A significant relationship was found between increased incidence of damage to peristomal skin and frequent removal of ostomy appliances, due to the stripping of the epidemal layer. ${ }^{[41]}$ The frequent removal of adhesive skin barriers increases the risk of physical damage to the stratum corneum. ${ }^{[49]}$ Changes in skin condition from frequent removal of skin barriers can be partially attributed to the adhesive power of the skin barriers. ${ }^{[41]}$ According to Omura, Yamabe, and Anazawa, ${ }^{[49]}$ as the adhesive power of the skin barrier increases, the incidence of peristomal skin changes increases. The authors found a correlation between the adhesive force of skin barriers and dermatologic changes $(p<.05)$, and this correlation is higher for papule, erosion, and pigmentation $(p<.01)$. Hydrocolloid skin barriers with high adhesive force increase the incidence of the flattening of the peristomal skin compared to skin barriers with less adhesive force $(74 \%$ vs. $58 \% ; p<.05) .{ }^{[51]}$ The high adhesive power of hydrocolloid skin barriers also increases the incidence of dermatological changes and roughens the peristomal skin more frequently than those with less adhesive force (26\% vs. $14 \%){ }^{[50]}$ Omura et al. ${ }^{[49]}$ stated that people who use skin barriers with an adhesive force of more than two Newton have increased incidence of dermatologic changes than those who use skin barriers with adhesive force of less than two Newton. It appears, therefore, frequent removal and higher adhesiveness of skin barrier have an association with the onset of PSCs.

\subsection{Demographic factors}

Demographic factors, such as age, gender, and BMI may also contribute to the development of PSCs. Jayarajah et al. ${ }^{[37]}$ reported that a certain age group have higher rate of complications. More specifically, patients younger than 60 years of age seem to encounter more skin irritation, greater leakage, and more difficulty in adjusting to life with an ostomy than the patients older than 80 years. ${ }^{[34]}$ In addition, the incidence of hyperplasia is also higher among patients younger than 65 years. ${ }^{[33]}$ However, some studies have found there is no relationship between age and development of PSCs. ${ }^{[32,41]}$

Both men and women are susceptible to developing PSCs, although differences in type and frequency were observed be- 
tween genders. According to Jayarajah et al. ${ }^{[37]}$ an increased incidence of PSCs in general was found among males. However, Ratliff ${ }^{[36]}$ has found that leakage was four times greater among women than in men. Likewise, the incidence of irritant contact dermatitis, hyperplasia, and peristomal hernias were also more frequently observed in women than in men. ${ }^{[33]}$ Sung et al. ${ }^{[33]}$ stated that the reason for a greater incidence of peristomal hernia is due to women's weaker abdominal muscle. However, not all researchers were in agreement with the gender differences in PSCs. For instance, Nybæk et al. ${ }^{[32]}$ reported in their study that there is no relationship between gender and development of PSCs ( $p=$ .826).

The occurrence of PSCs seems to be also related to BMI. Evidence showed that patients with a BMI greater than 30 had more skin complications such as early skin excoriation than patients with a BMI of less than $30 .^{[32]}$ In addition, the incidence of irritant contact dermatitis, hyperplasia, and peristomal hernias were also significantly higher in overweight and obese ostomates. ${ }^{[33]}$ Obesity leads to retraction or creasing of stomas, which creates a challenge in fitting ostomy appliances properly and contributes to the formation of irritant dermatitis. ${ }^{[4]}$ However, similar to findings related to gender and age, the impact of BMI on developing PSCs is conflicting. For example, Persson et al., Salvadalena; and Pittman et al. ${ }^{[33,38,40]}$ did not find a significant relationship between abnormal BMI and PSCs.

\section{Discussion}

The purpose of this integrative literature review is to explore risk factors associated with the development of PSCs in ostomy patients. The results of this review revealed seven thematic risk factors associated with PSCs: leakage of stoma output, type and structure of stoma, stoma site marking and the nature of surgery, ostomy education, ostomy appliances, mechanical trauma, and demographic factors.

One of the main findings of this study is the effect of stoma leakage on the increased risk of developing PSCs. The incidence of PSCs is increased when watery and corrosive ostomy effluent comes in contact with peristomal skin. ${ }^{[30,32,33,50]}$ These results are similar to the findings of previous studies, which showed that frequent exposure of peristomal skin to ostomy output is one of the main causes of the development of PSCs. ${ }^{[13,50]}$ Kwiatt and Kawata ${ }^{[50]}$ reported that the severity of chemical injury is dependent on the nature of stomal output and the duration of skin exposure to the effluent. Proteolytic enzymes present in the content of stoma output can cause redness, irritation, and peristomal skin breakdown. ${ }^{[51]}$ Prolonged exposure of stoma output compromises adhesiveness of skin barrier and results in breakdown of peristomal skin. ${ }^{[52]}$ Therefore, the incidence of PSCs is largely attributed to the effect of moisture from leakage of stoma output.

The type and structure of the stoma influenced the probability of contact between stoma output and skin, increasing the onset of PSCs. The higher incidence of PSCs observed among patients with ileostomy in this study has previously been reported. Carlsson, Fingren, Hallén, Petersen, and Lindholm $^{[53]}$ also found that patients with ileostomy showed a higher risk of developing PSCs in comparison to patients with urostomy or colostomy. The liquid and corrosive nature of the bilious small intestinal output and enzymes present in the ileostomy output damage the protein present in the outer layer of skin. ${ }^{[40,54]}$ In addition, Szymanski, St-Cyr, Alam, and Kassouf ${ }^{[55]}$ found that urostomy is the second most common type of stoma that contributes to the development of PSCs. The authors reported that prolonged contact with alkali present in the urine facilitates the alkaline encrustation in the peristomal skin of urostomates. The stool via colostomy is more formed and less acidic than with ileostomy or urostomy. ${ }^{[56]}$

Conflicting evidence between loop and end stomas was found in the result of this review. Some of the studies found more skin problems among individuals with loop stomas and others found more skin problems among individuals with end stomas. Doctor and Colibaseanu ${ }^{[25]}$ reported that individuals with loop stomas have higher incidence of PSCs. More specifically, loop ileostomy is the main cause of the development of peristomal skin excoriation because of the higher enzyme and watery content present in the proximal loop of ileum. ${ }^{[9,57]}$ However, there was no data found to support the prevalence of PSCs related to end stomas.

The findings of this study revealed the relationship between the structure of ostomies and PSCs. It was found that the shape and height of the stoma contribute to the development of PSCs. This finding is consistent with previous studies. Carlsson et al. ${ }^{[53]}$ stated that prevalence of PSCs is mainly related to the construction of ostomies. Doctor and Colibaseanu $^{[25]}$ reported that in general, insufficient protrusion and a structure of loop stoma that is too flat lead to pooling of ostomy effluent in the stoma site and the development of PSCs. Kann ${ }^{[40]}$ and Vujnovich ${ }^{[54]}$ found that poorly shaped stomas and low stomal height are factors that contribute to developing peristomal skin excoriation. As stated by Cottam, Richards, Hasted, and Blackman, ${ }^{[58]}$ as the height of stoma increases the rate of development of PSCs decreases. Evidence suggests that stoma height should be more than $1 \mathrm{~cm}$ in order to fix the stoma appliances properly. ${ }^{[40]}$ However, it is not clear what the exact height of the stoma should be. 
If stomas are at the skin level, this condition facilitates leakage and frequent removal of pouches, which, in turn, causes peristomal skin irritation. Not only is the height related to PSCs, the shape of the stoma is also a factor in the development of PSCs. Vujnovich ${ }^{[54]}$ found that an ideal spout of the stoma is necessary to prevent skin excoriation, especially with ileostomies and urostomies. If the spouts of stomas are not long enough, corrosive effluent from stomas will leak under the appliances and cause skin excoriation. ${ }^{[54]}$ It appears that length of spout is dependent on the height of the stoma.

Individuals who have not had stoma marking prior to surgery showed increased rates of PSCs in this study. ${ }^{[43,44]}$ Comprehensive plans for preventing risk of development of PSCs begin preoperatively by successful marking of the stoma site. This factor reduces potential issues related to proper stoma management, leakage, and the development of PSCs. The rate of PSCs is high in individuals without stoma marking. The negative effect of not having pre-operative stoma marking and incidence of PSCs was noted in the results of other studies. In a study conducted by Baykara et al. ${ }^{[59]}$ the rate of peristomal skin problems was significantly higher during emergency stoma surgery when the stoma site was not marked preoperatively compared to surgery when the stoma site was marked. If stoma marking were done preoperatively, the stoma would be created in an ideal location. $\mathrm{Kann}^{[40]}$ reported that the ideal location for a stoma is within the rectus abdominal muscle. These authors suggested that the stoma site should be in a flat area and away from scars, skin creases, and bony prominences. Kwiatt and Kawata ${ }^{[50]}$ found poorly located stomas resulted in ill fit of appliances, leakage of effluent, trauma, poor visualization of the stoma, and skin irritation, all of which further cause frustration, psychological distress, poor body image, and difficulty with postoperative adjustment in patients.

In planned ostomy surgery, the preparation time allow for a stoma marking, whereas in emergency surgery the preparation time is lacking. Therefore, evidence indicates that elective surgery is not associated with an overall increase of PSCs. Peristomal skin complication is associated with an increased likelihood of poor location of the stoma. Poor stoma site leads to stomas being created between skin creases, near bony prominences, or near incision lines, and contributes to related pouching problems.

The need for pre- and post-operative education by skilled WOCN, which plays a vital role in the management and prevention of stoma-related issues, was identified in this study. Previous studies reported that lack of regular follow- up in stoma clinics and stoma education are important contributing factors for peristomal dermatitis because of the high incidence of patients' inability to correctly fit ostomy appliances. ${ }^{[43]}$ Effective education includes stoma care, early identification of normal and abnormal peristomal skin, and the changing of ostomy appliances; this education prevents the occurrence of leakage and skin excoriation. ${ }^{[60]}$ Martins et al. ${ }^{[61]}$ found proper education and guidance helps individuals to perform proper stoma management and identify early signs of PSCs. These authors stated that some of the possible benefits include reduced treatment cost and improved ostomy patient QOL.

This study illustrated the importance of using proper appliances to prevent peristomal skin damage for individuals with a stoma. Convex appliances can increase or decrease the risk of developing PSCs. Buckle ${ }^{[62]}$ reported that pressure exerted from convex appliances increase the risk of developing peristomal skin erythema, bruising, or ulceration. However, Carlsson et al. ${ }^{[53]}$ found that in situations where the stoma is low in height, convex ostomy appliances could provide the opposite effect and prevent leakage of effluent by allowing the stoma to protrude further. Thus, a pouch with convexity can prevent risk of leakage and skin excoriation in flush or retracted stomas. ${ }^{[13]}$ The highest rate of leakage was associated with the flat appliances in comparison to convex appliances. In contrast, more redness and skin irritation of skin was found in patients using convex products compared to flat appliances. ${ }^{[63]}$ More research is needed to ascertain and verify reasons for PSCs related to different types of ostomy appliances.

Frequent removal of skin barriers increases the development of PSCs. The association between mechanical trauma and incidence of PSCs was evident in this review. Other studies have found a similar result of this review. Vujnovich ${ }^{[54]}$ found that frequent change of skin barriers causes the protective layer of the skin to be stripped off, which contributes to the development of skin excoriation. The author also stated that quick removal of appliances without supporting the abdominal skin as well as vigorous rubbing of skin develops skin problems such as denudation or ulceration, which leads to weeping or bleeding. Skin stripping due to removal of skin barriers causes separation of the epidermis from the dermis and deeper skin cell removals. ${ }^{[18,64]}$ This leads effluent from stoma, bacteria, and adhesive barrier ingredients to come into contact with the skin. Therefore, patients should wear a pouching system for between three to seven days while avoiding frequent removal of skin barriers. ${ }^{[65]}$

This study's finding that demographic factors are connected to the risk of developing PSC is consistent with the findings of previous studies in regard to patients' age and BMI. Respondents in Cottam et al.'s ${ }^{[58]}$ study who had experienced 
PSCs were younger than those who had not experienced PSCs. In contrast, however, another study revealed that advancing age is associated with increased incidence of skin problems. This may be due to the thinning of the epidermis and the expansion of the epidermal layer into the dermis to promote anchoring of the two skin layers because of an increase in age. ${ }^{[?]}$ Some previous studies found no differences between patients' age and development of PSCs. ${ }^{[66]}$ BMI is another demographic factor which increases the risk of the development of PSCs. Previous studies have reported that presence of excessive amounts of abdominal fat and skin folds leads to the formation of very low construction of stomas or constructions of stoma in abdominal creases. This diminishes visualization and interferes with management of the stoma, which leads to difficulty in fitting stoma appliances around body folds. Ill-fitting appliances increase the risk of developing PSCs due to the leakage of effluent. ${ }^{[50]}$ Gender also influences the risk of developing PSCs. The results of this study show that PSCs are more common in women than men. This is inconsistent with the findings of Taneja et al. ${ }^{[66]}$ who found a lower incidence of PSC in women. The existing evidence is insufficient to support the relationship between gender and PSCs in a conclusive direction.

\subsection{Limitations and recommendations}

The results of this integrative literature review need to be viewed in light of several limitations of the study. Most of the studies used newly established instruments for data collection without providing the necessary information on psychometric properties of the measures. Therefore, it is unknown whether the tools are reliable or valid. The convenience sampling method used in the studies was another limitation noted in this review. The convenience sampling method limits further generalizability of the findings due to the biased selection process. In addition, small sample sizes of study participants were noted in many of the studies included. Due to the paucity of studies, this review included studies of ostomy patients not only with CRC, but also with other health issues, which may represent a biased picture of risk factors related to CRCs. Some studies in this review used self-reported data by ostomy care experts, which may distort the results of the study. In addition, data obtained are limited to information from patients' medical records via retrospective chart review. Thus, the data obtained may include insufficient important information. More studies using interviews, focus groups, and questionnaires to collect data are necessary to identify results that are more accurate.

Other limitations of this review are related to study design. In the literature search, no studies focusing on the exclu- sive risk factors for the development of PSCs were found. Therefore, further studies are needed to better identify factors that influence the development of PSCs. Most of the studies included in this review used quantitative research methodology. Qualitative studies are needed to explore experiences of ostomy patients suffering from PSCs and to identify risk factors from the patients' perspective. No studies have been conducted in Qatar on the risk factors for the development of PSCs, and only one study has been conducted in the Middle East. This is another noteworthy limitation as there is a gap in knowledge regarding risk factors for developing PSCs in Arabic culture; as a result, the results of this study may not be directly generalizable to Qatar.

It would be interesting to compare the contributing factors of PSCs in Arabic countries to contemporary Western contexts. Further research is needed to examine specifically whether risk factors associated with PSCs among CRC patients in Qatar differ from patients in other countries. Future research should include both qualitative and quantitative methodologies involving the population in Qatar to explore reasons for the development of PSCs following ostomy surgery in CRC.

\subsection{Implications for practice}

Findings of this review suggest that precautions should be taken to prevent PSCs during the pre- and post-operative periods. Prior to all ostomy surgeries, proper pre-operative stoma site selection should be made to prevent leakage of effluent, reduce the risk of developing PSCs, and increase proper adherence of appliances. Many complications affecting peristomal skin require proper ostomy appliances to prevent leakage and protect skin. Proper appliances are required to maintain adequate wear time of the appliances, which reduces the development of peristomal skin excoriation. Therefore, health professionals should be educated on how to assess individuals at risk of developing PSCs as well as ostomy care in general, especially peristomal skin care. This would reduce treatment cost, decrease occurrence of PSCs, increase patients' knowledge regarding ostomy care, improves standard of care, and increase QOL of ostomy patients and families. Thus, early systematic and clientcentered training for health care providers about the proper ostomy care practices should be provided in the hospital and the community. This is necessary to maximize patients' self-mastery in ostomy care and overcome barriers to engage in effective management. In addition, it is very important to design, develop, and implement comprehensive educational programs for all clients with ostomies, as well as their families, about self-care, early recognition, and prevention of PSCs. Furthermore, regular follow-up care in a stoma clinic is necessary to increase patients' ability to care for an 
ostomy independently and would result in fewer peristomal skin-related problems.

Development of best nursing practice guidelines regarding ostomy care, especially peristomal skin care, by skilled ostomy care health professionals is necessary to assess ostomy care policies and practices, and to ensure ongoing opportunities to reinforce the importance of evidence- based practice. Support groups may be beneficial to assist ostomy patients to live with ostomy without skin complications especially in terms of peristomal skin care management.

Based on the identified clinical and physiological risk factors for the development of PSCs, in Qatar it is essential to develop and implement structured educational programmes for nurses as well as, for patients. This education will help in inculcating values and sense of responsibilities in patients for better ostomy care, early identification of the presence of complications, and to foster the practice of stoma care. Thus, in Qatar the findings of the review can be used as a basis of in-service education programs for nurses so as to make them aware of the present risk factors for developing PSC's. Furthermore, it is essential to have more multilingual CNS's to provide proper ostomy education because Qatar is a country with multicultural population. Also, in Qatar, it is more convenient to have different range of ostomy care products rather than having specific items to improve quality of patient care. Moreover, this review will motivate health professionals in Qatar to conduct further research. Further substantive research is required to validate the impact of effective education on improved outcomes related to peristomal skin care in Qatar.

\section{Conclusion}

This integrative literature review revealed that anyone with a stoma is at risk of developing PSCs. Peristomal skin requires special attention to decrease complications and minimize its effect. This focused review makes important contributions to the evidence related to risk factors of PSCs, and its results may help health professionals plan for effective prevention and management of PSCs. Comprehensive information regarding peristomal skin may potentially lead to the development of preventive and interventional strategies that will improve care and QOL for individuals living with stomas. Examination of the prevalence and severity of PSCs and the factors that contribute to the development of such complications expand scientific knowledge and provide a foundation upon which to build future research. This systematic review provides the best available evidence to develop evidence-based recommendations related to the risk factors associated with PSCs. While there is increasing evidence to support recommendations in some cases, it is clear that there remain gaps in the literature related to risk factors associated with PSCs, and areas in need of further research exist.

\section{CONFLICTS OF INTEREST Disclosure}

The authors declare that there is no conflict of interest.

\section{REFERENCES}

[1] Ferlay J, Soerjomataram I, Dikshit R, et al. Cancer incidence and mortality worldwide: Sources, methods and major patterns in GLOBOCAN 2012. International Journal of Cancer. 2015; 136(5): 359-386. PMid:25220842 https://doi.org/10.1002/ijc. 29210

[2] Bener A, Ayub H, Kakil R, et al. Patterns of cancer incidence among the population of Qatar: A worldwide comparative study. Asian Pacific Journal of Cancer Prevention. 2008; 9(1): 19-24.

[3] Sivaraman SK, Bhat AA, Prabhu KS, et al. Pristimerin Inhibits Growth and Induces Apoptosis in Human Colorectal Cancer Cells Through the Generation of Reactive Oxygen Species. In Qatar Foundation Annual Research Conference Proceedings. 2016.

[4] Gulf times. High incidence' of colon cancer among Qatari population. 2013.

[5] Hicks K, Sammo W, Huton D. Stoma care. In G. Nandakumar (Eds.), Evidence Based Practices in Gastrointestinal \& Hepatobiliary Surgery. JP Medical Ltd.; 2017.

[6] Meisner S, Lehur PA, Moran B, et al. Peristomal skin complications are common, expensive, and difficult to manage: A population based cost modeling study. PloS One. 2012; 7(5): e37813.

[7] Ay A, Bulut H. Assessing the validity and reliability of the peristomal skin lesion assessment instrument adapted for use in Turkey. Ostomy Wound Management. 2015; 61(8): 26-34.
[8] Bafford AC, Irani JL. Management and complications of stomas Surgical Clinics of North America. 2013; 93(1): 145-166. PMid:23177069 https://doi.org/10.1016/j.suc.2012.09. 015

[9] Yeo H, Abir F, Longo WE. Management of parastomal ulcers. World Journal of Gastroenterology. 2006; 12(20): 3133-3137. https : //doi.org/10.3748/wjg.v12.i20.3133

[10] Burch J. Care of patients with peristomal skin complications. Nursing Standard. 2014a; 28(37): 51-57. https ://doi.org/10.7748/ns .28.37.51.e8317

[11] Boyd K. Innovations in care: Managing severely excoriated peristomal skin using a hairdryer. Gastrointestinal Nursing. 2014; 12(10): 21-28. https://doi.org/10.12968/gasn.2014.12.10.21

[12] Colwell JC, Beitz J. Survey of wound, ostomy and continence (WOC) nurse clinicians on stomal and peristomal complications: A content validation study. Journal of Wound Ostomy \& Continence Nursing. 2007; 34(1): 57-69. https : //doi.org/10.1097/00152192-200 701000-00010

[13] Alvey B, Beck DE. Peristomal dermatology. Clinics in Colon and Rectal Surgery. 2008; 21(01): 041-044. PMid:20011395 https : //doi.org/10.1055/s-2008-1055320

[14] Watson AJ, Nicol L, Donaldson S, et al. Complications of stomas: their aetiology and management. British Journal of Community Nurs- 
ing. $2013 ; 18(3): 11-116$.

[15] Ratliff CR. Early peristomal skin complications reported by WOC nurses. Journal of Wound Ostomy \& Continence Nursing. 2010; 37(5): 505-510. PMid:20736859 https://doi .org/10.1097/WO N. 0b013e3181edac57

[16] Erwin-Toth P, Thompson SJ, Davis JS. Factors impacting the quality of life of people with an ostomy in North America: Results from the Dialogue Study. Journal of Wound Ostomy \& Continence Nursing. 2012; 39(4): 417-422. PMid:22652937 https: //doi.org/10.1097/WON.0b013e318259c441

[17] Herlufsen P, Olsen AG, Carlsen B, et al. Study of peristomal skin disorders in patients with permanent stomas. British Journal of Nursing 2006; 15(16): 854-862.

[18] Black P. Peristomal skin care: An overview of available products. British Journal of Nursing. 2007; 16(17): 1048-56. PMid:18026049 https://doi.org/10.12968/bjon.2007.16.17.27249

[19] Gooszen AW, Geelkerken RH, Hermans J, et al. Quality of life with a temporary stoma. Diseases of the Colon \& Rectum. 2000; 43(5): 650-655. https://doi.org/10.1007/BF02235581

[20] Holzer B, Matzel K, Schiedeck T, et al. Do geographic and educational factors influence the quality of life in rectal cancer patients with a permanent colostomy? Diseases of the Colon \& Rectum. 2005; 48(12): 2209-2216. PMid:16228820 https ://doi.org/10.1007/ s10350-005-0194-6

[21] Krouse R, Grant M, Ferrell B, et al. Quality of life outcomes in 599 cancer and non-cancer patients with colostomies. Journal of Surgical Research. 2007; 138(1): 79-87. PMid:17196990 https: //doi.org/10.1016/j.jss.2006.04.033

[22] Ross L, Abild-Nielsen AG, Thomsen BL, et al. Quality of life of Danish colorectal cancer patients with and without a stoma. Supportive Care in Cancer. 2007; 15(5): 505-513. PMid:17103196 https://doi.org/10.1007/s00520-006-0177-8

[23] Smith DM, Loewenstein G, Rozin P, et al. Sensitivity to disgust, stigma, and adjustment to life with a colostomy. Journal of Research in Personality. 2007; 41(4): 787-803. PMid:17940585 https: //doi.org/10.1016/j.jrp.2006.09.006

[24] Dabirian A, Yaghmaei F, Rassouli M, et al. Quality of life in ostomy patients: A qualitative study. Patient Preference and Adherence. 2010; 5: 1-5.

[25] Doctor K, Colibaseanu DT. Peristomal skin complications: Causes, effects, and treatments. Chronic Wound Care Management and Research. 2016; 4: 1-6. https://doi.org/10.2147/CWCMR.S936 15

[26] Nybaek H, Knudsen DB, Laursen TN, et al. Quality of life assessment among patients with peristomal skin disease. European Journal of Gastroenterology \& Hepatology. 2010; 22(2): 139-143. PMid:19918185 https ://doi.org/10.1097/MEG.0b013e3283 2ca054

[27] Torraco RJ. Writing integrative literature reviews: Guidelines and examples. Human Resource Development Review. 2005; 4(3): 356-367. https://doi.org/10.1177/1534484305278283

[28] Russell CL. An overview of the integrative research review. Progress in Transplantation. 2005; 15(1): 8-13. https ://doi.org/10.117 $7 / 152692480501500102$

[29] Whittemore R, Knafl K. The integrative review: updated methodology. Journal of Advanced Nursing. 2005; 52(5): 546-553. PMid:16268861 https://doi.org/10.1111/j.1365-2648.20 $05.03621 . \mathrm{x}$

[30] Burch J. Current nursing practice by hospital-based stoma specialist nurses. British Journal of Nursing. 2014b; 23: 31-34. PMid:24642773 https://doi.org/10.12968/bjon.2014.23.Sup5.S31
[31] McMullen CK, Hornbrook MC, Grant M, et al. The greatest challenges reported by long-term colorectal cancer survivors with stomas. Journal of Support Oncology. 2008; 6(4): 175-182.

[32] Nybaek H, Knudsen DB, Laursen TN, et al. Skin problems in ostomy patients: A case-control study of risk factors. Acta Dermatovenereologica. 2009; 89(1): 64-67. PMid:19197544 https : //doi. org/10.2340/00015555-0536

[33] Sung YH, Kwon I, Jo S, et al. Factors affecting ostomy-related complications in Korea. Journal of Wound Ostomy \& Continence Nursing 2010; 37(2): 166-172. PMid:20228659 https://doi.org/10.1 097/WON . Ob013e3181cf7b76

[34] Pittman J, Rawl SM, Schmidt CM, et al. Demographic and clinical factors related to ostomy complications and quality of life in veterans with an ostomy. Journal of Wound Ostomy \& Continence Nursing. 2008; 35(5): 493-503. PMid:18794701 https: //doi.org/10.1097/01.WON.0000335961.68113.cb

[35] Møller Kruse T, Storling ZM. Considering the benefits of a new stoma appliance: A clinical trial. British Journal of Nursing. 2015; 24: S12 S18. https://doi.org/10.12968/bjon.2015.24.Sup22.S12

[36] Ratliff CR. Factors related to ostomy leakage in the community setting. Journal of Wound Ostomy \& Continence Nursing. 2014; 41(3): 249-253. PMid:24503813 https://doi .org/10.1097/WO N. 0000000000000017

[37] Jayarajah U, Samarasekara AM, Samarasekera DN. A study of longterm complications associated with enteral ostomy and their contributory factors. BMC Research Notes. 2016; 9(1): 500. PMid:27919277 https://doi.org/10.1186/s13104-016-2304-z

[38] Lindholm E, Persson E, Carlsson E, et al. Ostomy-related complications after emergent abdominal surgery: A 2-year follow-up study. Journal of Wound Ostomy \& Continence Nursing. 2013; 40(6): 603 610. PMid:24108321 https://doi.org/10.1097/WON.0b013e 3182a9a7d9

[39] Persson E, Berndtsson I, Carlsson E, et al. Stoma-related complications and stoma size-a 2-year follow up. Colorectal Disease. 2010; 12(10): 971-976. PMid:19519689 https ://doi.org/10.1111/j . 1463-1318.2009.01941.x

[40] Kann BR. Early stomal complications. Clinics in Colon and Rectal Surgery. 2008; 21(01): 023-030. PMid:20011393 https : //doi .or $\mathrm{g} / 10.1055 / \mathrm{s}-2008-1055318$

[41] Salvadalena GD. The incidence of stoma and peristomal complications during the first 3 months after ostomy creation. Journal of Wound Ostomy \& Continence Nursing. 2003; 40(4): 400-406. PMid:23820472 https ://doi.org/10.1097/WON.0b013e3182 $95 \mathrm{a} 12 \mathrm{~b}$

[42] Williams J, Gwillam B, Sutherland N, et al. Evaluating skin care problems in people with stomas. British Journal of Nursing. 2010; 19(17): S6-S15

[43] Colwell JC, McNichol L, Boarini J. North America wound, ostomy, and continence and enterostomal therapy nurses current ostomy care practice related to peristomal skin issues. Journal of Wound, Ostomy, and Continence Nursing. 2017; 44(3): 257-261. PMid:28362656 https://doi.org/10.1097/WON.0000000000000324

[44] Millan M, Tegido M, Biondo S, et al. Preoperative stoma siting and education by stomatherapists of colorectal cancer patients: A descriptive study in twelve Spanish colorectal surgical units. Colorectal Disease. 2010; 12(7): e88-e92.

[45] Alenezi AN, Mansour EA. Impact of stoma care education in minimizing the incidence of stoma skin complications. Bahrain Medical Bulletin. 2016; 38(3): 151-153. https://doi.org/10.12816/0 047489

[46] McMullen CK, Wasserman J, Altschuler A, et al. Untreated peristomal skin complications among long-term colorectal cancer sur- 
vivors with ostomies: Lessons from a study of family caregiving. Clinical Journal of Oncology Nursing. 2011; 15(6): 644650. PMid:22119975 https://doi.org/10.1188/11.CJON. 64 4-650

[47] Coloplast. Ostomy pouching systems. 2013. Available from: https://www. coloplast.us/Global/US/Ostomy/Professi onal/Wellness\%20Articles/Pouching\%20Systems.pdf

[48] Szewczyk MT, Majewska G, Cabral MV, et al. The effects of using a moldable skin barrier on peristomal skin condition in persons with an ostomy: results of a prospective, observational, multinational study. Ostomy/Wound Management. 2014; 60(12): 16-26.

[49] Omura Y, Yamabe M, Anazawa S. Peristomal skin disorders in patients with intestinal and urinary ostomies: influence of adhesive forces of various hydrocolloid wafer skin barriers. Journal of Wound Ostomy \& Continence Nursing. 2010; 37(3): 289-298. PMid:20436373 https : //doi .org/10.1097/WON . 0b013e3181 d8c9d3

[50] Kwiatt M, Kawata M. Avoidance and management of stomal complications. Clinics in Colon and Rectal Surgery. 2013; 26(2): 112-121. PMid:24436659 https://doi .org/10.1055/s-0033-1348050

[51] Gray M, Black JM, Baharestani MM, et al. Moisture-associated skin damage: Overview and pathophysiology. Journal of Wound Ostomy \& Continence Nursing. 2011; 38(3): 233-241. PMid:21490547 https://doi.org/10.1097/WON.0b013e318215f798

[52] Cutting K. Comparing ostomates' perceptions of hydrocolloid and silicone seals: a survey. British Journal of Nursing. 2016; 25(22): S24S29. https://doi.org/10.12968/bjon.2016.25.22.S24

[53] Carlsson E, Fingren J, Hallén AM, et al. The prevalence of ostomyrelated complications 1 year after ostomy surgery: A prospective, descriptive, clinical study. Ostomy/Wound Management. 2016; 62(10): 34-48.

[54] Vujnovich A. The management of stoma-related skin complications. WOUNDS UK. 2006; 2(3): 36-47.

[55] Szymanski KM, St-Cyr D, Alam T, et al. External stoma and peristomal complications following radical cystectomy and ileal conduit diversion: a systematic review. Ostomy/Wound Management. 2010; 56(1): 28-35.

[56] Husain SG, Cataldo TE. Late stomal complications. Clinics in Colon and Rectal Surgery. 2008; 21(01): 031-040. PMid:20011394 https://doi.org/10.1055/s-2008-1055319
[57] Patil V, Vijayakumar A, Ajitha MB, et al. Comparison between tube ileostomy and loop ileostomy as a diversion procedure. ISRN Surgery. 2012; $1-5$.

[58] Cottam J, Richards K, Hasted A, et al. Results of a nationwide prospective audit of stoma complications within 3 weeks of surgery. Colorectal Disease. 2007; 9(9): 834-838. PMid:17672873 https : //doi.org/10.1111/j.1463-1318.2007.01213.x

[59] Baykara ZG, Demir SG, Karadag A, et al. A multicenter, retrospective study to evaluate the effect of preoperative stoma site marking on stomal and peristomal complications. Ostomy Wound Management. 2014; 60(5): 16-26.

[60] Burch J. Stoma management: Enhancing patient knowledge. British Journal of Community Nursing. 2011; 16(4): 162-166. PMid:21471916 https://doi.org/10.12968/bjcn.2011.16. 4.162

[61] Martins L, Tavernelli K, Sansom W, et al. Strategies to reduce treatment costs of peristomal skin complications. Gastrointestinal Nursing. $2012 ; 10(10)$

[62] Buckle N. The dilemma of choice: Introduction to a stoma assessment tool. Gastrointestinal Nursing. 2013; 11(4): 26-32. https: //doi.org/10.12968/gasn.2013.11.4.26

[63] Redmond C, Cowin C, Parker T. The experience of faecal leakage among ileostomists. British Journal of Nursing. 2009; 18(6): S12 S17.

[64] Stelton S, Zulkowski K, Ayello EA. Practice implications for peristomal skin assessment and care from the 2014 World Council of Enterostomal Therapists International Ostomy Guideline. Advances in Skin \& Wound Care. 2015; 28(6): 275-284. PMid:25988737 https://doi.org/10.1097/01.ASW.0000465374.42350.0f

[65] Colwell JC, Ratliff CR, Goldberg M, et al. MASD part 3: Peristomal moisture-associated dermatitis and periwound moisture-associated dermatitis: A consensus. Journal of Wound Ostomy \& Continence Nursing. 2011; 38(5): 541-553. PMid:21873913 https://doi .or g/10.1097/WON. 0b013e31822acd95

[66] Taneja C, Netsch D, Rolstad BS, et al. Clinical and Economic Burden of Peristomal Skin Complications in Patients With Recent Ostomies. Journal of Wound, Ostomy, and Continence Nursing. 2017; 44(4): 350-357. PMid:28574928 https://doi .org/10.1097/wO N. 0000000000000339 